\title{
Phase portrait of Hamiltonian systems with homogeneous nonlinearities*
}

\author{
Armengol Gasull \\ Dept. de Matemàtiques \\ Universitat Autònoma de Barcelona. \\ Edifici Cc 08193 Bellaterra, Barcelona. Spain. \\ gasull@mat.uab.es, \\ Antoni Guillamon \\ Dept. de Matemàtica Aplicada I \\ Universitat Politècnica de Catalunya. \\ Dr. Marañón 44-50, 08028 Barcelona. Spain. \\ toni@ma1.upc.es \\ Víctor Mañosa ${ }^{\dagger}$ \\ Dept. de Matemàtica Aplicada III \\ Universitat Politècnica de Catalunya. \\ Colom 1, 08222 Terrassa, Barcelona. Spain. \\ manosa@ma3.upc.es
}

May 3, 1999

\begin{abstract}
The main goal of this work is to describe the phase portrait of Hamiltonian systems with a non degenerate center at the origin and homogeneous nonlinearities of arbitrary degree $n$. We apply our results to the case $n=2$ to re-obtain the bifurcation diagram of all the quadratic Hamiltonian vector fields with a centre at the origin, as well as to the case $n=3$.
\end{abstract}

Keywords: bifurcations, Hamiltonian system, phase portrait, period annulus.

\footnotetext{
${ }^{*}$ Partially supported by the DGICYT grant number PB96-1153.
}

$\dagger$ Corresponding author. 


\section{Introduction}

In this paper we study the phase portraits of Hamiltonian systems of the form

$$
\left\{\begin{array}{l}
\dot{x}=-H_{y}(x, y), \\
\dot{y}=H_{x}(x, y),
\end{array}\right.
$$

where $H(x, y)=\frac{x^{2}+y^{2}}{2}+H_{n+1}(x, y)$, and $H_{n+1}$ is a homogeneous polynomial of degree $n+1$. The solutions of system (1) are contained in the level curves $\{H(x, y)=h, h \in$ $\mathbb{R}\}$. Furthermore, the origin is a non degenerate centre.

The knowledge of phase portraits of Hamiltonian systems is useful for several reasons. We comment some of them in the sequel.

(i) Small polynomial perturbation of them give raise to polynomial systems with limit cycles. For instance, the cubic system with 11 limit cycles presented in [1] uses this idea. Therefore the knowledge of the number and distribution of centres that a Hamiltonian system can have gives information about the number of limit cycles and the way in which they can be nested for general polynomial planar systems.

(ii) For Hamiltonian systems or systems which can be transformed to them (like the Lotka-Volterra one), a problem which is frequently studied is the problem of the variation of the period of the closed orbits in terms of the energy, see for instance [2] or [3] and the references therein. The simplest case, also of interest (see [4]), is the one in which the period of the orbits is constant for all the period annulus of the center (which for us will be the largest neighbourhood of the center entirely covered by periodic orbits.) In this case, the critical point is called an isochronous center and as it has already been noticed (for instance in [4]), isochronous centres can only exist if the period annulus of the center is unbounded. Therefore, for some families of Hamiltonian systems, the fact that all their period annulus are bounded will prevent the existence of isochronous centres (this will be the case of our family (1) with $n$ even). See [2] and [5] again.

(iii) The knowledge of the phase portrait of Hamiltonian systems of the form $H(x, y)=\frac{(F(x, y))^{2}+(G(x, y))^{2}}{2}$, where $F$ and $G$ are polynomials such that $\frac{\partial F}{\partial x} \frac{\partial G}{\partial y}-\frac{\partial F}{\partial y} \frac{\partial G}{\partial x} \equiv$ 1 , will prove or disprove the celebrated Jacobian Conjecture in $\mathbb{R}^{2}$ proposed by O. Keller in 1939, see [6] and [7].

Other questions are addressed just to better understand planar polynomial Hamiltonian systems of degree $n$. Among the others we want to recall these two questions (see [8] and [9]): Which is the maximum number of centres that such systems can have in terms of $n$ ? Which kind of boundaries can have the period annulus of Hamiltonian centres? Both questions are solved in this paper for the special family (1), see Proposition 1 in next section. 
Our procedure to obtain the phase portrait of (1) is different from previous approaches used for $n=2$, see [10] or [11]. It is presented in Section 3 as an algorithm which allows to depict the phase portrait of any system of type (1) in terms of the shape of $g(\theta)=H_{n+1}(\cos \theta, \sin \theta)$. Furthermore, this approach allows to get the full bifurcation diagram of (1) when there are involved some parameters $\Lambda$ in it, in terms of the bifurcation diagram of their associated function $g_{\Lambda}(\theta)$. The bifurcation diagram of $g_{\Lambda}(\theta)$ is made taking into account their zeroes, maxima, minima, inflexion points, and the value of $g_{\Lambda}(\theta)$ at these points, as will be explained in Sections 3 and 4. We also will see that the bifurcation diagram of the function $g_{\Lambda}(\theta)$ contains in general more bifurcation surfaces that the one of the system (1) because there are different shapes of $g_{\Lambda}(\theta)$ which produce the same phase portrait for (1), see Remark 6 in Section 3.3.

We want to stress that our approach to describe the phase portrait reminds the study of conservative systems with one degree of freedom, that is Hamiltonian systems with Hamiltonian function of type $H(x, y)=\frac{y^{2}}{2}+V(x)$. For those systems, one can determine the dynamics of the system from the observation of the behaviour of the potential function $V(x)$, see [12] for instance, as we determine the dynamics of (1) from $g(\theta)$.

As an application of our algorithm, in Section 4 we obtain all the phase portraits of (1) for $n=2$ and $n=3$, as well as two bifurcation diagrams: the complete one for $n=2$ and the one corresponding to reversible systems of type (1) for $n=3$. We have already commented that the case $n=2$ is well-understood ([10] and [11]) but as far as we know the case $n=3$ was not yet studied. The result that we obtain can be inscribed inside a collection of papers trying to get all phase portraits for some particular polynomial families of ordinary differential equations, see for instance [13], [14], [15], or [16].

To end this introduction, we would like to explain that, when a first version of this paper was ended, the authors presented it as a poster in the "Symposium on Planar Vector Fields" that took place in Lleida in November 1996. The lecture that D. Schlomiuk gave there about an algebro-geometric way to characterize bifurcation diagrams (see [11]) motivated us to enlarge the first version of our work including an analysis of bifurcations (Section 4), with the aim of showing the role of $g(\theta)$ in them.

Next section is devoted to prove the results on the type of singularities (finite and infinite) of (1) in a suitable compactification. These results are the key part of the paper.

\section{Statements and proofs of the main results.}

Before stating our results we introduce a variation of the Poincaré compactification. 


\subsection{Compactification of the system.}

In order to get a complete description of the topological behaviour of the flow in a neighbourhood of an infinite or a finite critical point, we will use a compactification in which the global phase portrait is similar to the one on the Poincarés disk, see [17] and [18], and which is inspired in [19] and [14].

Bringing system (1) to polar coordinates we obtain:

$$
\left\{\begin{array}{l}
\dot{r}=-g^{\prime}(\theta) r^{n} \\
\dot{\theta}=1+(n+1) g(\theta) r^{n-1}
\end{array}\right.
$$

where $g(\theta)=H_{n+1}(\cos \theta, \sin \theta)$ is a homogeneous trigonometrical polynomial of degree $n+1$. This system is defined on the cylinder $C=\mathbb{R}^{+} \times S^{1}=\left\{(r, \theta): r \in \mathbb{R}^{+}, \theta \in\right.$ $\left.S^{1}=\mathbb{R} / 2 \pi \mathbb{Z}\right\}$.

We make the following transformation in order to simplify (2):

$$
R=\frac{r^{n-1}}{1+r^{n-1}}
$$

In this way, system (2) writes as

$$
\left\{\begin{aligned}
\dot{R} & =-(n-1) R^{2} g^{\prime}(\theta) \\
\dot{\theta} & =1+(n+1) \frac{R}{1-R} g(\theta)
\end{aligned}\right.
$$

Observe that the circle $(0, \theta)$ has been mapped onto itself, while the infinity has been mapped onto the circle given by $R=1$. To avoid the singularity on $R=1$ in the second equation, we make the change of time:

$$
\frac{d t}{d \tau}=1-R
$$

We continue calling the derivatives with respect to $\tau$ as $\dot{x}$ and $\dot{y}$. With this new time the system is transformed into

$$
\left\{\begin{aligned}
\dot{R} & =-(n-1) R^{2}(1-R) g^{\prime}(\theta)=: F(R, \theta), \\
\dot{\theta} & =1+R((n+1) g(\theta)-1)=: G(R, \theta) .
\end{aligned}\right.
$$

Observe that $R=0$ is always an invariant circle. Undoing the changes of variables, this circle is transformed into the centre at the origin. We are interested in the phase portrait of (4) just in the disk $\{R \leq 1\}$. The interior of this disk, $\{R<1\}$, will be called the finite part and (4) the modified polar compactification of (1). In this region there are critical points $(R, \theta)$, which will be called finite critical points, when $g^{\prime}(\theta)=0, g(\theta)<0$ and $R=\frac{1}{1-(n+1) g(\theta)}$. When $R=1$, the critical points $(1, \theta)$ must satisfy $g(\theta)=0$; we call them infinite critical points. 


\subsection{Main results in the classification of finite and infinite critical points.}

Finite critical points of planar Hamiltonian systems are well-known. In fact, their indices, which satisfy $i \leq 1$, classify the type of critical point. More concretely, if the index is 1 (resp. $i<1$ ) then the critical point is a center (resp. a critical point with $2|i|+2$ hyperbolic sectors). Next result is a particularization of the general result for systems of type (1).

Theorem 1 For systems of type (1), there are only three possible types of finite critical points: centre points, saddle points (4 hyperbolic sectors), and those with 2 hyperbolic sectors. Moreover, if $p=\left(r_{*}, \theta_{*}\right)$ is a critical point, then $g\left(\theta_{*}\right)<0$, $g^{\prime}\left(\theta_{*}\right)=0, r_{*}^{n-1}=\frac{-1}{(n+1) g\left(\theta_{*}\right)}$ and

a) $p$ is a centre if and only if $\theta_{*}$ is a local maximum of $g(\theta)$.

b) $p$ is a saddle-point if and only if $\theta_{*}$ is a local minimum of $g(\theta)$.

c) $p$ is the union of two hyperbolic sectors if and only if $\theta_{*}$ is an inflexion point of $g(\theta)$.

As we will see in next subsection the proof of the generic case $\left(g^{\prime \prime}\left(\theta^{*}\right) \neq 0\right)$ is trivial, while the proof in the general case takes more computations and is based on the use of the modified polar compactification and on the classification result of degenerate singularities due to Andreev (see [20]). We remark that in the usual polar coordinates or in the cartesian coordinates the proof of the result would be much more difficult.

Infinite critical points in the Poincaré compactification are more complicated. In [8] and [2] they are studied and, among other things, it is proved that hyperbolic sectors with finite separatrices are not very frequent. In the next theorem we classify them in the modified polar compactification of system (1) in terms of $g$.

Theorem 2 Suppose that $q$ is an infinite critical point of the modified polar compactification system (1), associate to the critical direction $\theta_{*}$. Then, $q$ has one and only one sector in the finite part which is

a) elliptic if and only if $\theta_{*}$ is a local maximum of $g(\theta)$.

b) hyperbolic if and only if $\theta_{*}$ is a local minimum of $g(\theta)$. Moreover, the separatrices of this sector lay on $R=1$.

c) parabolic repelling (resp. attracting) if and only if $g(\theta)$ is monotonously increasing (resp. decreasing) in a neighbourhood of $\theta_{*}$. 
In the proof of the above theorem the coordinates introduced in the generalized polar compactification play again a key role.

A consequence of Theorems 1 and 2 is the following result.

Proposition 1 The following statements hold.

i) The maximum number of centres for a system of type (1) is $n / 2+1$ (resp $n+2)$ when $n$ is even (resp. odd).

ii) The origin of (1) is either a global centre or has a bounded period annulus. Furthermore, it is a global center if and only if $g(\theta) \geq 0$ (and so $n$ is odd). A centre $p$ of (1) different from the origin has a bounded period annulus.

iii) Given a centre of system (1) with bounded period annulus, the maximum number of critical points in the boundary of its period annulus is $n / 2+1$, if $n$ is even, and either infinity or $n+1$, if $n$ is odd. Furthermore, there are infinitely many critical points only when $n$ is odd and $g(\theta)=H_{n+1}(\cos \theta, \sin \theta)$ is a negative constant.

Moreover, all the upper bounds stated above are the sharpest ones.

\subsection{Proof of Theorem 1.}

The linear part of $(4)$ at a finite critical point $p=\left(R_{*}, \theta_{*}\right)$ is:

$$
\left(\begin{array}{cc}
0 & \frac{(n+1)(n-1) g\left(\theta_{*}\right) g^{\prime \prime}\left(\theta_{*}\right)}{\left(1-(n+1) g\left(\theta_{*}\right)\right)^{3}} \\
(n+1) g\left(\theta_{*}\right)-1 & 0
\end{array}\right)
$$

Thus, we easily get:

Proposition 2 If $p=\left(R_{*}, \theta_{*}\right)$ is a critical point of (4), with $0<R_{*}<1$, such that $g^{\prime \prime}\left(\theta_{*}\right)<0$ (resp., $g^{\prime \prime}\left(\theta_{*}\right)>0$ ), then $p$ is a centre (resp., a saddle point) for the system.

To study the case in which $g^{\prime \prime}\left(\theta_{*}\right)=0$, we will apply a classification result due to Andreev (see [20]), stated in the Appendix.

We apply the change of time $\left(\tau^{\prime}\right.$ will be the new variable):

$$
\frac{d \tau}{d \tau^{\prime}}=\frac{1}{(n+1) g\left(\theta_{*}\right)-1}
$$


which is well-defined because $g\left(\theta_{*}\right)<0$, but reverses the sense of the flow. After this change, equation (4) turns into

$$
\left\{\begin{aligned}
\dot{R} & =\frac{-(n-1) R^{2}(1-R) g^{\prime}(\theta)}{(n+1) g\left(\theta_{*}\right)-1}=: \tilde{F}(R, \theta) \\
\dot{\theta} & =\frac{1+R((n+1) g(\theta)-1)}{(n+1) g\left(\theta_{*}\right)-1}=: \tilde{G}(R, \theta) .
\end{aligned}\right.
$$

Lemma 1 Let $\varphi_{j, k}$ denote the partial derivative $\frac{\partial^{j+k} \varphi}{\partial R^{j} \partial \theta^{k}}$ of a function $\varphi(R, \theta)$ at a critical point $p=\left(R_{*}, \theta_{*}\right)$. Then, the functions $\tilde{F}(R, \theta)$ and $\tilde{G}(R, \theta)$ appearing in (6) satisfy:

a)

$$
\begin{aligned}
\tilde{F}_{m, 0} & =0, m \geq 1, \quad \tilde{F}_{0,1}=\frac{-(n+1)(n-1) g g^{\prime \prime}}{(1-(n+1) g)^{4}} \\
\tilde{F}_{0, m} & =\frac{-\left(n^{2}-1\right) g g^{(m+1)}}{(1-(n+1) g)^{4}}, \quad \tilde{F}_{1, m-1}=\frac{-(n-1) g^{(m)}(1+2(n+1) g)}{(1-(n+1) g)^{3}}, \\
\tilde{F}_{2, m-1} & =\frac{-(n-1) g^{(m)}(4+2(n+1) g)}{(1-(n+1) g)^{2}}, \quad \tilde{F}_{3, m-1}=\frac{-6(n-1) g^{(m)}}{(1-(n+1) g)}, m \geq 2, \\
\tilde{F}_{k, m-k} & =0, k \geq 4, m \geq k+1 .
\end{aligned}
$$

b)

$$
\begin{aligned}
\tilde{G}_{1,0} & =1, \quad \tilde{G}_{0,1}=0, \\
\tilde{G}_{m, 0} & =0, \quad \tilde{G}_{0, m}=\frac{-(n+1) g^{(m)}}{(1-(n+1) g)^{2}}, m \geq 2, \\
\tilde{G}_{1, m-1} & =\frac{-(n+1) g^{(m-1)}}{1-(n+1) g}, m \geq 2, \quad \tilde{G}_{k, m-k}=0, k \geq 2 .
\end{aligned}
$$

where $g:=g\left(\theta_{*}\right), g^{\prime}:=g^{\prime}\left(\theta_{*}\right), \ldots, g^{(k)}:=g^{(k)}\left(\theta_{*}\right)$.

The proof of the above lemma follows from straightforward derivations and substitutions at $p$.

The proof of Theorem 1 follows directly from the next result.

Lemma 2 For systems of class (6), there are only three possible types of finite critical points: centre points, saddle points (4 hyperbolic sectors), and those with 2 hyperbolic sectors. Moreover, if $p=\left(R_{*}, \theta_{*}\right)$ is such a critical point, then $g\left(\theta_{*}\right)<0, g^{\prime}\left(\theta_{*}\right)=0$, $R_{*}=\left(1-(n+1) g\left(\theta_{*}\right)\right)^{-1}$ and 
a) $p$ is a centre if and only if $\theta_{*}$ is a local maximum of $g\left(\theta_{*}\right)$.

b) $p$ is a saddle-point if and only if $\theta_{*}$ is a local minimum of $g\left(\theta_{*}\right)$.

c) $p$ is the union of two hyperbolic sectors if and only if $\theta_{*}$ is an inflexion point of $g\left(\theta_{*}\right)$.

Proof of Lemma 2. Applying Lemma 1, we can write system (6) using the series expansions of $\tilde{F}(R, \theta)$ and $\tilde{G}(R, \theta)$. After a rotation if necessary, we can take $\theta_{*}=0$. In these new variables, the critical point $p=\left(R_{*}, \theta_{*}\right)$ writes as $p=\left(R_{*}, 0\right)$, where $R_{*}=\frac{1}{1-(n+1) g(0)}$.

Suppose now that $g^{\prime}(0)=g^{\prime \prime}(0)=\ldots=g^{(m)}(0)=0$ and $g^{(m+1)}(0) \neq 0$, for $m \geq 2$. Then, performing also the translation $\rho=R-R_{*}$,

$$
\left\{\begin{aligned}
\dot{\rho}= & \frac{-\left(n^{2}-1\right) g(0) g^{(m+1)}(0)}{m !(1-(n+1) g(0))^{4}} \theta^{m}+\frac{-(n-1) g^{(m+1)}(0)(1+2(n+1) g(0))}{m !(1-(n+1) g(0))^{3}} \rho \theta^{m}+ \\
& \frac{-\left(n^{2}-1\right) g(0) g^{(m+2)}(0)}{(m+1) !(1-(n+1) g(0))^{4}} \theta^{m+1}+o(m+1) \\
\dot{\theta}= & \rho+\frac{-(n+1) g^{(m+1)}(0)}{(m+1) !(1-(n+1) g(0))^{2}} \theta^{m+1}+ \\
& \frac{-(n+1) g^{(m+1)}(0)}{(m+1) !(1-(n+1) g(0))} \rho \theta^{m+1}+\frac{-(n+1) g^{(m+2)}(0)}{(m+2) !(1-(n+1) g(0))^{2}} \theta^{m+2}+o(m+2) .
\end{aligned}\right.
$$

By the sake of simplicity, some times we will write it as:

$$
\left\{\begin{array}{l}
\dot{\rho}=\alpha_{0, m} \theta^{m}+\alpha_{1, m} \rho \theta^{m}+\alpha_{0, m+1} \theta^{m+1}+o(m+1)=: X_{1}(\rho, \theta), \\
\dot{\theta}=\rho+\beta_{0, m+1} \theta^{m+1}+\beta_{1, m+1} \rho \theta^{m+1}+\beta_{0, m+2} \theta^{m+2}+ \\
o(m+2)=: X_{2}(\rho, \theta) .
\end{array}\right.
$$

Notice that $\alpha_{0, m} \beta_{0, m+1} \neq 0$ since we are under the hypotheses $g(0)<0$ and $g^{(m+1)}(0) \neq 0$. It is clear that the former critical point is now the origin of this system and that the linear part at it is given by the nilpotent matrix $\left(\begin{array}{ll}0 & 0 \\ 1 & 0\end{array}\right)$. For this kind of points, we can apply the classification result stated in the Appendix.

First of all, we must express the solution of $X_{2}(\rho, \theta)=0$ as $\rho=\rho(\theta)$. Then, we must compute $\Phi_{1}(\theta)=X_{1}(\rho(\theta), \theta)$ and $\Phi_{2}(\theta)=\left(\frac{\partial X_{1}}{\partial \rho}+\frac{\partial X_{2}}{\partial \theta}\right)(\rho(\theta), \theta)$.

If we put $\rho(\theta)=\sum_{k} c_{k} \theta^{k}$ in $X_{2}=0$, it is easy to see that we must have $c_{j}=0$, for all $j=0, \ldots, m$. Thanks to this observation and the kind of powers that appear in $X_{2}$, we can avoid the computation of the other $c_{j}$. 
Then,

$$
\begin{aligned}
\Phi_{1}(\theta) & =\alpha_{0, m} \theta^{m}+\alpha_{1, m}\left(\sum_{k} c_{k} \theta^{k}\right) \theta^{m}+\alpha_{0, m+1} \theta^{m+1}+o(m+1)= \\
& \alpha_{0, m} \theta^{m}+\alpha_{0, m+1} \theta^{m+1} \alpha_{1, m} c_{m+1} \theta^{2 m+1}+o(2 m+1)= \\
\Phi_{2}(\theta)=\left(\alpha_{1, m}+(m+1) \beta_{0, m+1}\right) \theta^{m}+\left(2 \alpha_{2, m}+(m+1) \beta_{1, m+1}\right) \rho(\theta) \theta^{m}+ & \left(\alpha_{1, m+1}+(m+2) \beta_{0, m+2}\right) \theta^{m+1}+o(m+1)= \\
& \left(\alpha_{1, m}+(m+1) \beta_{0, m+1}\right) \theta^{m}+\left(\alpha_{1, m+1}+(m+2) \beta_{0, m+2}\right) \theta^{m+1}+ \\
& o(m+1)= \\
& \left(\alpha_{1, m}+(m+1) \beta_{0, m+1}\right) \theta^{m}+o(m) .
\end{aligned}
$$

Hence, according to the notation used in the Andreev's Theorem (see the Appendix), we set $K=\alpha_{0, m}, \kappa=m$ and $\lambda \geq k$.

In the case that $m$ is odd, $\theta=0$ is either a local maximum or a local minimum of $g(\theta)$; if $g^{m+1}(0)>0$-that is, a minimum of $g$ - then we have $K>0$ and, since $\kappa$ is an odd number, by Andreev's Theorem 2$),\left(R_{*}, 0\right)$ is a topological saddle. If $g^{m+1}(0)<0$ -that is, a maximum of $g$ - then we have $K<0$, and since $\kappa$ is an odd number with $\kappa<2 \lambda+1$, then again by Andreev's Theorem 3c), the origin is a either a focus or a centre; however, since (1) is Hamiltonian, it cannot be a focus and so we have that $\left(R_{*}, 0\right)$ is a centre.

In the case that $m$ is even, since $\kappa<2 \lambda+1$, by Andreev's Theorem 1b), $\left(R_{*}, 0\right)$ is a cusp.

\subsection{Proof of Theorem 2.}

For the infinite critical points $p=\left(1, \theta_{*}\right)$ of $(4)$, where $g\left(\theta_{*}\right)=0$, we have that the Jacobian matrix is:

$$
\left(\begin{array}{cc}
(n-1) g^{\prime}\left(\theta_{*}\right) & 0 \\
-1 & (n+1) g^{\prime}\left(\theta_{*}\right)
\end{array}\right) .
$$

As in the finite case, the generic situation $g^{\prime}\left(\theta_{*}\right) \neq 0$ is very easy. In fact, it is an attracting node if $g^{\prime}\left(\theta_{*}\right)<0$, and a repelling one if $g^{\prime}\left(\theta_{*}\right)>0$.

The difficulties arise when $g^{\prime}\left(\theta_{*}\right)=0$. Then, the linear part writes as $\left(\begin{array}{cc}0 & 0 \\ -1 & 0\end{array}\right)$, and we can use the same technique of the finite case. The results and proofs are also similar:

Lemma 3 Let $\varphi_{j, k}$ denote the partial derivative $\frac{\partial^{j+k} \varphi}{\partial R^{j} \partial \theta^{k}}$ of a function $\varphi(R, \theta)$ at a critical point $p=\left(R_{*}, \theta_{*}\right)$. Then, the functions $F(R, \theta)$ and $G(R, \theta)$ appearing in (6) satisfy: 
a)

$$
\begin{array}{ll}
F_{1, m}=(n-1) g^{(m+1)}, & F_{2, m}=4(n-1) g^{(m+1)} \\
F_{3, m}=6(n-1) g^{(m+1)}, & F_{m, 0} \equiv 0, m \geq 4 \\
F_{0, m} \equiv 0, m \geq 1 . &
\end{array}
$$

b)

$$
\begin{aligned}
& G_{1,0}=-1, \quad G_{1, m}=(n+1) g^{(m)}, m \geq 1, \\
& G_{m, 0} \equiv 0, m \geq 2, \quad G_{0, m}=(n+1) g^{(m)}, m \geq 1,
\end{aligned}
$$

where $g:=g\left(\theta_{*}\right), g^{\prime}:=g^{\prime}\left(\theta_{*}\right), \ldots, g^{(k)}:=g^{(k)}\left(\theta_{*}\right)$.

The proof of Theorem 2 follows directly from the following result.

Lemma 4 Suppose that $(R, \theta)=\left(1, \theta_{*}\right)$ is an infinite critical point for the system (4), and assume also that $g\left(\theta_{*}\right)=g^{\prime}\left(\theta_{*}\right)=\ldots=g^{(m)}\left(\theta_{*}\right)=0$ and $g^{(m+1)}\left(\theta_{*}\right) \neq 0$, = with $m \geq 0$. Then, the critical point has one and only one sector in the finite part which is

a) elliptic if and only if $m$ is odd and $g^{(m+1)}\left(\theta_{*}\right)<0$.

b) hyperbolic if and only if $m$ is odd and $g^{(m+1)}\left(\theta_{*}\right)>0$. Moreover, the separatrices of this sector lay on the circle $R=1$.

c) parabolic - in fact the critical point has a repelling node (resp. an attracting node) in the finite part- if and only if $m$ is even and $g^{(m+1)}\left(\theta_{*}\right)<0$ (resp. $\left.g^{(m+1)}\left(\theta_{*}\right)>0\right)$.

Proof of Lemma 4. By rotation, if necessary, we can take $\theta_{*}=0$ (the critical point, now, is $p=(1,0))$ and, by the translation $R-1=\rho$, the critical point becomes $(\rho, \theta)=(0,0)$. We also reverse the sign of the time by $\frac{d \tau}{d \tau^{\prime}}=-1$, in order to have the standard nilpotent linear part at the critical point.

Suppose now that $g(0)=g^{\prime}(0)=g^{\prime \prime}(0)=\ldots=g^{(m)}(0)=0$ and $g^{(m+1)}(0) \neq 0$. As for the finite points, using Lemma 3 and series expansions of $F(R, \theta)$ and $G(R, \theta)$, we obtain:

$$
\left\{\begin{aligned}
\dot{\rho} & =-\frac{n-1}{m !} g^{(m+1)} \rho \theta^{m}-\left(\frac{n-1}{(m+1) !} g^{(m+2)} \rho \theta^{m+1}\right. \\
& \left.+\frac{2(n-1)}{m !} g^{(m+1)} \rho^{2} \theta^{m}\right)+o(m+2)=: X_{1}(\rho, \theta), \\
\dot{\theta} & =\rho-\frac{(n+1)}{(m+1) !} g^{(m+1)} \theta^{m+1}-\frac{(n+1)}{(m+1) !} g^{(m+1)} \rho \theta^{m+1} \\
& -\frac{(n+1)}{(m+2) !} g^{(m+2)} \theta^{m+2}+o(m+2)=: X_{2}(\rho, \theta) .
\end{aligned}\right.
$$


Following the same notation as in (8), we also note that the first coefficient of $X_{1}(\rho, \theta)$ does not vanish, $\alpha_{1, m}=-\frac{n-1}{m !} g^{(m+1)} \neq 0$, since we are under the hypothesis $g^{(m+1)}(0) \neq 0$. We will apply again the classification criterion for this kind of critical points.

Analogously to the finite case, we put $\rho(\theta)=\sum_{k} c_{k} \theta^{k}$ in $X_{2}=0$. It turns out that

$$
\rho(\theta)=\frac{n+1}{(m+1) !} g^{(m+1)} \theta^{m+1}+o(m+1) .
$$

Then,

$$
\begin{aligned}
& \Phi_{1}(\theta)=-\frac{\left(g^{(m+1)}\right)^{2}\left(n^{2}-1\right)}{(m+1) ! m !} \theta^{2 m+1}+o(2 m+1) \\
& \Phi_{2}(\theta)=-\frac{2 n}{m !} g^{(m+1)} \theta^{m}+o(m)
\end{aligned}
$$

The first non-vanishing term of $\Phi_{1}(\theta)$ has odd degree and negative coefficient.

So according with the notation used in Andreev's Theorem, we set $K=$ $-\frac{\left(g^{(m+1)}\right)^{2}\left(n^{2}-1\right)}{m !(m+1) !}<0, \kappa=2 m+1, L=-\frac{2 n}{m !} g^{(m+1)}(0)$, and $\lambda=m$. Observe that $\kappa=2 \lambda+1$ and $L^{2}+4 K(\lambda+1)=\frac{4\left(g^{(m+1)}(0)\right)^{2}}{(m !)^{2}}>0$.

In the case that $m$ is odd, $\theta=0$ is either a local maximum or a local minimum of $g(\theta)$; since $\lambda$ is odd, $\kappa=2 \lambda+1$ and $L^{2}+4 K(\lambda+1)>0$, then by Andreev's Theorem the critical point is formed by the union of an hyperbolic sector and an elliptic sector. We just must take care of the sign to ensure whether the hyperbolic sector or the elliptic sector lays on the finite part.

In the case that $m$ is even, $\theta=0$ is an inflexion point of $g(\theta)$; since $\lambda$ is odd, $\kappa=2 \lambda+1$ and $L^{2}+4 K(\lambda+1)>0$, we have by statement 3a of Andreev's Theorem that, if $g^{m+1}(0)>0$ then $L<0$, and the critical point is an stable node (remember that we have reversed the time and so the point is actually an unstable node for the original system); and, if $g^{m+1}(0)<0$ then $L>0$, and the critical point is an unstable node (so a stable node for the original system).

Now we prove the last part of statement (b).

The separatrices of the hyperbolic sector are not finite since otherwise the description of the separatrices obtained applying Andreev's Theorem would be in contradiction with the description of the separatrices which go to infinity given in Theorem 2.2 (ii) of [8] and in [2].

\subsection{Proof of Proposition 1.}

Proof of Proposition 1. Let us prove (i). Since $g(\theta)$ is a homogeneous trigonometric polynomial of degree $n+1$, in $[0,2 \pi)$ there are at most $2 n+2$ oscillations of $g(\theta)$ and, 
since $g(\theta+\pi)=(-1)^{n+1} g(\theta)$, if $n$ is even at most $n+1$ oscillations can be negative (indeed, at most $n / 2$ of them can be maxima). So, including the origin, system (1) can have at most $n / 2+1$ centres. Otherwise, if $n$ is odd, all the $2 n+2$ oscillations of $g(\theta)$ can be negative. So, there can exist at most $n+1$ negative maxima of $g(\theta)$. In this situation, system (1) would have at most $n+2$ centres.

Now we prove (ii). A centre $p$ cannot have an unbounded but not global period annulus since this implies the existence of a hyperbolic sector at the infinity having at least one finite separatrix, which is in contradiction with Theorem 2 (b). Furthermore, notice that from Theorem 1, the origin is a global centre of (1) if and only if $g(\theta)=$ $H_{n+1}(\cos \theta, \sin \theta) \geq 0$, and this can only occur when $n$ is odd.

To prove statement (iii), we recall that if $g(\theta)$ is a homogeneous trigonometric polynomial of degree $n+1$, there can be at most $2 n+2$ oscillations of $g(\theta)$ in $[0,2 \pi)$. Using the same argument as in (i), we have that if $n$ is even, at most $n+1$ oscillations can be negative (indeed, at most $n / 2+1$ of them can be on the same level curve of $g(\theta))$. If $n$ is odd, all the $2 n+2$ oscillations of $g(\theta)$ can be negative. So, there can exist at most $n+1$ negative oscillations of $g(\theta)$ on the same level curve.

It is easy to see that, from equation (2), there are infinitely many critical points if and only if $g(\theta) \equiv C<0$ (and this can only occur if $n$ is odd). In this case, all the points of the circle defined by $r^{n-1}=\frac{-1}{(n+1) C}$ are critical points.

\section{An algorithm to depict the phase portrait.}

\subsection{Preliminary questions.}

This section is devoted to obtain a useful algorithm in order to depict the phase portrait of (1). We intend to face the practical side of the problem.

The knowledge of the Hamiltonian on some special curves is crucial to study the phase portrait, and so we first stress some trivial properties concerning them:

Remark 1 Suppose that $g\left(\theta_{0}\right)=0$, for some $\theta_{0} \in[0,2 \pi)$. Then, $H\left(r, \theta_{0}\right)=\frac{1}{2} r^{2}$ and $\left.\dot{\theta}\right|_{\theta=\theta_{0}} \equiv 1$.

According to this remark, we define a basic sector as follows:

Definition 1 A region

$$
\Omega=\left\{(r, \theta): \theta_{m} \leq \theta \leq \theta_{M} ; g\left(\theta_{m}\right)=g\left(\theta_{M}\right)=0, g(\theta)<0 \text { for all } \theta \in\left(\theta_{m}, \theta_{M}\right)\right\},
$$

will be called a basic sector of the phase portrait of (2), see Fig. 1. 
Of course, on the two boundaries of a basic sector $\Omega, \theta=\theta_{m}$ and $\theta=\theta_{M}$ we will have infinite critical directions. In the sectors where $g(\theta)>0$, the vector field has no critical points and so, the flow is very easy to describe. In fact, in virtue of Remark 1 , if $g\left(\theta_{1}\right)=g\left(\theta_{2}\right)=0$ and $g(\theta)>0$ for all $\theta \in\left(\theta_{1}, \theta_{2}\right)$, then the Poincaré map between $\left\{\theta=\theta_{1}\right\}$ and $\left\{\theta=\theta_{2}\right\}$ defined by the flow and parameterized by $h$ is well-defined and onto.

We will represent the phase portrait on the disk $\{R \leq 1\}$. Its representation will be given separately on every basic sector (if there exist). In this case the complete description comes from joining all the basic sectors. Furthermore, when $n$ is odd, the function $g(\theta)$ has even symmetry and so, it suffices to analize the phase portrait for $\theta \in[0, \pi]$; when $n$ is even, $g(\theta)$ has odd symmetry and its better to analize it for $\theta \in[0,2 \pi]$.

Another important factor is the localization of the curve $\Theta_{0}:=\{(r, \theta): \dot{\theta}=0\}$ inside each basic sector. Directly from (2), we can deduce that

Remark 2 The curve $\Theta_{0}$ can be parameterized by $\theta$ as

$$
r(\theta)=\left(\frac{-1}{(n+1) g(\theta)}\right)^{1 /(n-1)} .
$$

It passes, of course, through every critical point and

$$
\lim _{\theta \rightarrow \theta_{m}^{+}} r(\theta)=\lim _{\theta \rightarrow \theta_{M}^{-}} r(\theta)=+\infty,
$$

see Fig. 2.

In the next lemma, we see several properties that will be very helpful in the description of the phase portrait:

Lemma 5 The Hamiltonian $H(r, \theta)$ restricted to the curve $\Theta_{0}$ is a positive-valued increasing function of $r(\theta)$ (in fact, it depends only on the value of $g(\theta)$ ). As a consequence, two points $\left(r_{i}, \theta_{i}\right)$, for $i=1,2$, situated on $\Theta_{0}$ belong to the same level curve if and only if $g\left(\theta_{1}\right)=g\left(\theta_{2}\right)$.

Proof. Substituting expression (10) into the expression of the Hamiltonian, we obtain:

$$
\begin{array}{cl}
H(r(\theta), \theta) & =\frac{1}{2} r(\theta)^{2}+r(\theta)^{n+1} g(\theta)=r(\theta)^{2}\left(\frac{1}{2}+r(\theta)^{n-1} g(\theta)\right)= \\
& r(\theta)^{2}\left(\frac{1}{2}-\frac{1}{n+1}\right)>0, \quad \text { since } n \geq 2 .
\end{array}
$$

Using this equation the proof is trivial.

From this lemma we will be able to decide, for instance, whether two finite saddles connect or not.

Finally, we establish the last preliminary result: 
Lemma 6 The Hamiltonian function restricted to a non critical direction $\theta=\theta_{*}$ has a unique maximum which is located at $\left\{\theta=\theta_{*}\right\} \cap \Theta_{0}$ and, moreover, $\lim _{r \rightarrow+\infty} H\left(r, \theta_{*}\right)=$ $-\infty$.

The knowledge of the topology of the separatrices of the saddles points and of the points with only two hyperbolic sectors determines the full phase portrait, see [21]. Next section deals with this problem.

\subsection{Study of saddle points and their separatrices.}

In this study, it will be important to distinguish between systems having basic sectors and systems not having them.

If the system does not contain basic sectors, this can be due to two facts:

- $g(\theta) \geq 0$ for all $\theta$

- $g(\theta)<0$ for all $\theta$.

In the first case, next discussion is not necessary because we do not have finite critical points. The second case can be treated using similar ideas than used in the present section.

Let $\Omega=\left\{(r, \theta): \theta_{m} \leq \theta \leq \theta_{M}\right\}$ be a basic sector. It is clear, by Remark 2, that the curve $\Theta_{0}$ divides $\Omega$ into two disjoint open subsets, $\Omega^{+}$and $\Omega^{-}$, such that $\overline{\Omega^{+} \cup \Omega^{-}}=\Omega$ and $\dot{\theta}>0$ in $\Omega^{+}$(resp., $\dot{\theta}<0$ in $\Omega^{-}$), see Fig. 2.

Lemma 7 In a neighbourhood of a saddle $s_{A} \in \Omega$, two of its separatrices (one stable and one unstable) lay in $\Omega^{+}$and the other two (one stable and one unstable) lay in $\Omega^{-}$.

Proof. We prove it only for $\Omega^{+}$. The other case is identical.

Take a saddle-point at $s=\left(r_{*}, \theta_{*}\right)$. Let $s(\theta)$ be a separatrix of $s$, which lays on $\Omega^{+}$at least for $\theta \in\left(\theta_{*}-\epsilon, \theta_{*}\right)$. Since $\dot{\theta}$ is positive, it must be a stable separatrix. By the same reason, there cannot be unstable separatrices of $s$ defined in $\Omega^{+}$for $\theta<\theta_{*}$. Similarly, the region of $\Omega^{+}$where $\theta>\theta_{*}$ cannot contain stable separatrices of $s$. Then, by the alternation of stable and unstable separatrices, there can only be one stable separatrix of $s$ and one unstable in $\Omega^{+}$.

Among the saddles points we will distinguish two behaviours, see also Fig. 3: 
Definition 2 Let $\Omega=\left\{(r, \theta): \theta_{m} \leq \theta \leq \theta_{M}\right\}$ be a basic sector and $s_{A}=\left(r_{A}, \theta_{A}\right) \in \Omega$ a saddle of this sector.

We say that $s_{A}$ fulfills property $\left(\mathbf{L S}^{-}\right)$, the lowest saddle from the left, if it satisfies $g\left(\theta_{A}\right)<g(\theta)$, for all $\theta \in\left(\theta_{m}, \theta_{A}\right)$.

Analogously, we say that $s_{A}$ fulfills property $\left(\mathbf{L S}^{+}\right)$, the lowest saddle from the right, if it satisfies $g\left(\theta_{A}\right)<g(\theta)$, for all $\theta \in\left(\theta_{A}, \theta_{M}\right)$.

If some saddle $s_{A}$ fulfills property $\left(\mathrm{LS}^{-}\right)$, then the stable separatrix lying on $\Omega^{+}$ must leave the basic sector for negative time, otherwise it would intersect the curve $\Theta_{0}$ in some point $s_{A^{\prime}}=\left(r_{A^{\prime}}, \theta_{A^{\prime}}\right)$ with $\theta_{A^{\prime}} \in\left(\theta_{m}, \theta_{A}\right)$. But, from Lemma 5 , this would imply that $g\left(\theta_{A^{\prime}}\right)=g\left(\theta_{A}\right)$, a contradiction with $\left(\mathrm{LS}^{-}\right)$. Therefore, the stable separatrix must intersect (for negative time) the line $\theta=\theta_{m}$. By similar arguments, on $\Omega^{-}$, the unstable separatrix leaves $s_{A}$ and dies at the infinite critical point on the direction $\theta=\theta_{m}$.

If it fulfills $\left(\mathrm{LS}^{+}\right)$, it can be deduced in an analogous way that the unstable separatrix of $s_{A}$ lying in $\Omega^{+}$must cut the line $\theta=\theta_{M}$, while the stable separatrix in $\Omega^{-}$ comes from the critical point on the direction $\theta=\theta_{M}$ of the infinity and goes to $s_{A}$.

Let us suppose now that property $\left(\mathrm{LS}^{-}\right)$is not fulfilled for some saddle then, we will prove that this yields the existence of some homoclinic or heteroclinic loop. Suppose then, that the saddle $s_{A}=\left(r_{A}, \theta_{A}\right)$ does not fulfill $\left(\mathrm{LS}^{-}\right)$, see saddle $s_{2}$ in Fig. 3. In this case, let $s_{A^{\prime}}$ be the closest saddle with $\theta_{A^{\prime}}<\theta_{A}$ that fulfills property $\left(\mathrm{LS}^{-}\right)$. Frow the above reasonings we know that there is a negatively invariant region $\mathcal{I}$, defined by $\theta=\theta_{M}$, the stable separatrix of $s_{A^{\prime}}$ coming from infinity, and the unstable one quitting the basic sector through $\theta=\theta_{M}$. One unstable separatrix of $s_{A}$ can leave $\mathcal{I}$ through $\theta=\theta_{M}$, but the other unstable one must remain inside this region; otherwise, Lemma 6 will be violated (in particular on $\theta=\theta_{M}$ ). Since the system is Hamiltonian, the unstable separatrix that remains in $\mathcal{I}$ must form either an homoclinic loop or an heteroclinic connection. The heteroclinic connection takes place if and only if there is another saddle than $s_{A}$ between $s_{A}$ and $s_{A^{\prime}}$ on the same energy level.

The symmetric situations are performed if property $\left(\mathrm{LS}^{+}\right)$is not fulfilled (formation of homoclinic or heteroclinic loops surrounding the left-closest centre).

Collecting all these remarks, we can reach the following algorithm to depict the phase portrait of a system of type (2).

\subsection{The algorithm.}

A) If $g(\theta) \geq 0$ for all $\theta$, then the origin is a global centre. 
B) If $g(\theta)<0$ for all $\theta$, then basic sectors are not defined. If $g(\theta)$ is constant, the phase portrait is a "global" centre but with a closed curve full of critical points. Otherwise, there exists a set $S=\left\{\theta_{1}<\ldots<\theta_{2 J}\right\}, J \geq 1$, of absolute minima of $g(\theta)$. Denote by $s_{k}=\left(r_{k}, \theta_{k}\right)$ the corresponding saddle points. Then, two separatrices of $s_{k}$ coincide with two separatrices of $s_{k-1}$, and the other two with two of $s_{k+1}$, for $k \in\{1, \ldots, 2 J\}\left(s_{2 J+1}:=s_{1}, s_{0}:=s_{2 J}\right)$. The phase portrait inside the $2 J$ invariant sets defined by above heteroclinic connections can be obtained following steps 3 and 4, taking into account that there are not saddle points satisfying $\left(L S^{-}\right)$or $\left(L S^{+}\right)$.

C) Otherwise:

1. Plot the curve $\Theta_{0}$. The local minima of this curve are saddle points, while the maxima are centre points.

2. All the separatrices of saddles which satisfy $\left(L S^{-}\right)$and $\left(L S^{+}\right)$leave (some times for negative times) the basic sector: one through $\theta=\theta_{m}$, another one through $\theta=\theta_{M}$ and those lying in $\Omega^{-}$go to the infinite critical points of the sector, see Fig. 3

3. Any saddle $s_{A}$ not satisfying $\left(L S^{-}\right)$has an homoclinic loop which also passes through the closest point $\left(r_{B}, \theta_{B}\right) \in \Theta_{0}$ in the same basic sector such that $g\left(\theta_{A}\right)=g\left(\theta_{B}\right)$ and $\theta_{B}<\theta_{A}$. If the new point is also a saddle point $s_{B}$, the loop becomes heteroclinic. This loop surrounds at least one centre region in between and, eventually, other homoclinic or heteroclinic loops as well as more centres.

4. Any saddle $s_{A}$ not satisfying $\left(L S^{+}\right)$has an homoclinic loop which also passes through the closest point $\left(r_{B}, \theta_{B}\right) \in \Theta_{0}$ such that $g\left(\theta_{A}\right)=g\left(\theta_{B}\right)$ and $\theta_{B}>\theta_{A}$. Again, if the new point is also a saddle point $s_{B}$, the loop becomes heteroclinic.

5. If there is some critical point with only two hyperbolic sectors, we can do the phase portrait as if these critical points do not exist and, afterwards, insert them at the corresponding places.

6. Fill all the regions in which $\{R<1\}$ is divided by the separatrices using the local behaviours of the critical points described in Theorems 1 and 2 .

Next remarks try to clarify some degenerate situations.

Remark 3 The finite critical points with two hyperbolic sectors can be understood as the merging of a finite saddle and a finite centre, see Fig. 4. The period annulus of the centre shrinks to a single point and so, two of the four hyperbolic sectors of the saddle are lost. 
Remark 4 The hyperbolic sectors at infinity can be thought as the merging of two infinite nodes and one finite saddle. At the limit, the nodes disappear and the inner separatrices of the saddle collapse to the infinity. Then, the presence of a hyperbolic sector at infinity does not alter the topological phase portrait, except for this point.

Remark 5 The points having an elliptic sectors at infinity can be obtained from a finite centre $c:=\left(r_{*}, \theta_{*}\right)$ moving to infinity, see Fig. 5, as if all the points $\left(r, \theta_{*}\right)$ with $r \geq r_{*}$ collapsed to the point at infinity corresponding to the direction $\theta=\theta_{*}$.

More precisely, the boundary of the elliptic sector with associated critical direction $\theta_{*}$, situated between two saddles $s_{i}=\left(r_{i}, \theta_{i}\right)$ and $s_{i+1}=\left(r_{i+1}, \theta_{i+1}\right)\left(\theta_{i}<\theta_{*}<\theta_{i+1}\right)$ is formed by:

a) two orbits going from $p$ to $s_{i}$ and from $s_{i}$ to $p$, if $g\left(\theta_{i}\right)<g\left(\theta_{i+1}\right)$.

b) two orbits going from $p$ to $s_{i+1}$ and from $s_{i+1}$ to $p$, if $g\left(\theta_{i}\right)>g\left(\theta_{i+1}\right)$.

c) three orbits going from $p$ to $s_{i}$, from $s_{i}$ to $s_{i+1}$ and from $s_{i+1}$ to $p$, if $g\left(\theta_{i}\right)=$ $g\left(\theta_{i+1}\right)$.

Remark 6 From the algorithm, it is clear that, given a $g(\theta)$, the key points to plot the phase portrait are their zeroes, extrema and inflexion points, and the values of $g(\theta)$ at them. We want to stress that $g(\theta)$ with different qualitative behaviours (in the previous sense) can give rise to the same phase portrait, see Fig. 6.

\section{Examples and applications of the algorithm.}

In this section we will apply the algorithm described in the previous section to obtain all the phase portraits for $n=2$ and $n=3$. We also want to discuss the bifurcation diagram in the quadratic case and in a particular family of cubic systems.

If we want to discuss the bifurcation diagram of a system of type (1) with associated non-trivial function $g(\theta)$ a $(k+1)$-parametric trigonometric polynomial of degree $n+1$ - that is we consider a family with $k \leq n+1$ varying coefficients-, notice that by using rescalings of the type $u=\lambda x, v=\lambda y$ the parameter space can be considered as $S^{k}$ - the $k$-dimensional sphere-. Observe that as $g(\theta)$ has degree $n+1$, if $n$ is even, this implies that the phase portraits of two opposite points in $S^{k}$ are equivalent and so the parameter space can be considered as $\mathbb{R} P(k)$ - the $k$-dimensional projective space-, that is: we only need to know the bifurcation diagram in a closed half-sphere of $S^{k}$. When $n$ is odd is necessary to study the whole sphere. 


\subsection{The Quadratic Case.}

We apply the algorithm presented in Section 3 to give a classification of quadratic systems of type (1), which is depicted in Fig. 7 (a more complete classification, including also those Hamiltonian systems without a critical point at the origin, can be found in [10]). In our case, the clue is to study the different possible shapes of $g(\theta)=H_{3}(\cos \theta, \sin \theta)$. For this classification, we follow the notation introduced in [22] and also used in [10], where the different phase portraits are called Vulpe- $j$, with $j \in J \subset \mathbb{N}$.

Moreover, it is also possible to obtain the bifurcation diagram for the Quadratic Hamiltonian Systems (QHS) with a critical point at the origin in terms of the variation of $g(\theta)$. We summarize this part in Theorem 3 and in Fig. 8.

\subsubsection{Classification of phase portraits.}

Since $g(\theta)$ is an odd trigonometric function, it must exist some point $\theta_{0}$ such that $g\left(\theta_{0}\right)=0$. We can always take $\theta_{0}$ in order that $g^{\prime}\left(\theta_{0}\right)<0$, except when $g\left(\theta_{0}\right)=$ $g^{\prime}\left(\theta_{0}\right)=g^{\prime \prime}\left(\theta_{0}\right)=0$, in which case the phase portrait is topologically equivalent to Vulpe-2. Accordingly, we consider the set

$$
G^{\prime}:=\left\{\theta \in\left(\theta_{0}, \theta_{0}+\pi\right): g^{\prime}(\theta)=0\right\} .
$$

In case that $\# G^{\prime}=3$, we assume that the three elements satisfy $\theta_{1}<\theta_{2}<\theta_{3}$. By (12), we know that $\operatorname{sign} g\left(\theta_{1}\right)=\operatorname{sign} g\left(\theta_{3}\right)=-1$, and so only the sign of $g\left(\theta_{2}\right)$ can change; we define $\sigma=\operatorname{sign} g\left(\theta_{2}\right)$ and $g_{i}=\left|g\left(\theta_{i}\right)\right|$, for $i=1,2,3$.

With this notation, we can state:

Proposition 3 Suppose that there exists a $\theta_{0}$ such that $g\left(\theta_{0}\right)=0$ and $g^{\prime}\left(\theta_{0}\right)<0$. Then, the phase portrait of a system (1), with $n=2$, is topologically equivalent to Vulpe-j (see Fig. 7), where

1. $j=2$, if $\# G^{\prime}=1$.

2. $j=7$, if $\# G^{\prime}=2$.

3. $j \in\{3,4,5,6,8,9,10,11\}$, if $\# G^{\prime}=3$. More precisely,

(a) $j=3$, if $\sigma=-1$ and $g_{1}=g_{3}$.

(b) $j=4$, if $\sigma=-1$ and $g_{1} \neq g_{3}$.

(c) $j=5$, if $\sigma=0$ and $g_{1}=g_{3}$.

(d) $j=6$, if $\sigma=0$ and $g_{1} \neq g_{3}$.

(e) $j=8$, if $\sigma=+1$ and $g_{i}=g_{j}>g_{k}$, with $\{i, j, k\}=\{1,2,3\}$. 
(f) $j=9$, if $\sigma=+1$ and $g_{i}=g_{j}<g_{k}$, with $\{i, j, k\}=\{1,2,3\}$.

(g) $j=10$, if $\sigma=+1$ and $g_{i}=g_{j}=g_{k}$, with $\{i, j, k\}=\{1,2,3\}$.

(h) $j=11$, if $\sigma=+1$ and $g_{i} \neq g_{j}$, for $i \neq j$.

If $\theta_{0}$ does not exist, then $j=2$.

\subsubsection{Bifurcation diagram.}

Observing Fig. 7 and also the conditions stated in Proposition 3, one can notice that Vulpe-4,11 are structurally stable systems inside the family (1), while Vulpe-3,6,7,8,9 have one degree of structural unstability and Vulpe-5,10, two degrees. Notice that Vulpe-2 can be either structurally stable or structurally unstable of codimension 2 . It is also evident that the structural unstability is produced by one of the three following facts:

- Two of the three critical points have the same absolute value of $g(\theta)$. As we have studied in Section 3.2, this fact leads to a connection between saddles.

- One of the critical points (in fact, $\theta_{2}$ in the above notation) is also a zero of $g(\theta)$. This means that a finite critical point is converted into an infinite critical point.

- One of the critical points has multiplicity two (the second derivative of $g(\theta)$ also vanishes). This represents a collision between a centre and a saddle, and gives a critical point with two hyperbolic sectors.

Of course, codimension-two bifurcations can only appear when two of these facts hold simultaneously.

Then, to obtain the bifurcation diagram, we must give the equations of the curves on which these codimension one bifurcations occur, in some set of parameters. To avoid cumbersome notation, we have choosen to depict the bifurcation diagram in terms of the coefficients of $g^{\prime}(\theta)$ instead of those of $g(\theta)$. We first notice the following fact:

Lemma 8 Given a trigonometric homogeneous function $g(\theta)$ of degree 3 , it is always possible to express its derivative as

$$
g^{\prime}(\theta)=\sin \theta\left(\alpha \cos ^{2} \theta+\beta \cos \theta \sin \theta+\gamma \sin ^{2} \theta\right)
$$

by means of a translation of the variable $\theta$. 
The proof of this lemma follows from the fact that, since $g(\theta)$ is of odd degree, it has always a local extremum (call it $\theta^{*}$ ), which can be moved to the origin by a change of type $\tilde{\theta}=\theta-\theta^{*}$.

Theorem 3 Consider the Hamiltonian systems which Hamiltonian function is $H_{3}(r, \theta)=\frac{1}{2} r^{2}+r^{3} g(\theta)$. Suppose that $g^{\prime}(\theta)=\sin \theta\left(\alpha \cos ^{2} \theta+\beta \cos \theta \sin \theta+\gamma \sin ^{2} \theta\right)$, and $\alpha, \beta, \gamma \in \mathbb{R}$, satisfying $\alpha^{2}+\beta^{2}+\gamma^{2} \neq 0$. When $\gamma \neq 0$, define $\tilde{\alpha}=\alpha / \gamma, \tilde{\beta}=\beta / \gamma$, $\Delta=\tilde{\beta}^{2}-4 \tilde{\alpha}$ and $T_{i}=\left(-\tilde{\beta}+(-1)^{i+1} \sqrt{\Delta}\right) / 2$.

Then, there appear bifurcations of codimension at most two inside this family of systems. More precisely, in the space $(\alpha, \beta, \gamma)$ :

1. The codimension two bifurcation points are the intersections of the codimension one curves, and are the following: $(\tilde{\beta}, \tilde{\alpha})=(0,0), \alpha=\gamma=0$ and $\beta=\gamma=0$ (Vulpe-2); $(\tilde{\beta}, \tilde{\alpha})=(0,-2),(\tilde{\beta}, \tilde{\alpha})=(-\sqrt{2},-4)$ and $(\tilde{\beta}, \tilde{\alpha})=(\sqrt{2},-4)$ (Vulpe5); and, $(\tilde{\beta}, \tilde{\alpha})=(0,-3)$ (Vulpe-10).

2. The codimension one curves are (except for the codimension two points stated above):

(a) $\alpha-\beta=\gamma=0, \alpha \cdot \beta \neq 0$ or $\alpha+\beta=\gamma=0, \alpha \cdot \beta \neq 0$, where a heteroclinic connection of saddles takes place (Vulpe-3);

(b) $\tilde{\alpha}>0$ and $-\left(\tilde{\alpha}+\tilde{\beta} T_{i}\right)\left(\Delta-\tilde{\alpha}^{2}\right)-\tilde{\alpha}^{2}=0$, for $i=1,2$, where a heteroclinic connection of saddles takes place (Vulpe-3);

(c) $\tilde{\alpha}<0$, and $\tilde{\beta}=0$ or $-\left(\tilde{\alpha}+\tilde{\beta} T_{i}\right)\left(\Delta-\tilde{\alpha}^{2}\right)-\tilde{\alpha}^{2}=0$, for $i=1,2$, where a heteroclinic connection of saddles takes place (Vulpe-3,8 or 9), and the sequence on each of these curves is, in some parameterization, Vulpe-3-58-10-9;

(d) $\tilde{\alpha}=-2$ or $\tilde{\beta}^{2}=-4 /(2+\tilde{\alpha})$, where degenerate critical points at infinity appear (Vulpe-6);

(e) $\tilde{\alpha}=0$ or $\Delta=0$, where a finite critical point with two hyperbolic sectors is formed (Vulpe- 7 ).

3. The rest of points in the bifurcation space correspond to structurally stable systems. In particular, the systems are topologically equivalent to Vulpe- $j$, with

(a) $j=4$, if $\gamma=0$, not satisfying statements (1) or (2).

(b) $j=2$, if $\Delta<0$.

(c) $j=4$, if $\Delta>0$ and $\tilde{\alpha}>-2$, or $\tilde{\alpha}<-2\left(2+\tilde{\beta}^{2}\right) / \tilde{\beta}^{2}$, not satisfying statements (1) or (2).

(d) $j=11$ if $-2\left(2+\tilde{\beta}^{2}\right) / \tilde{\beta}^{2}<\tilde{\alpha}<-2$, not satisfying statements (1) or (2). 
This theorem describes completely all the bifurcations and can be abridged in the diagram given in Fig. 8.

Proof of Theorem 3: We only give the sketch of the proof, avoiding the straightforward computations.

The main step consists of finding the curves where the codimension one happens. This question can be analysed by solving the equations:

1. $g(0)=0$.

2. $g\left(\theta_{i}\right)=0$, where $\tan \theta_{i}=: T_{i}=\left(-\tilde{\beta}+(-1)^{i+1} \sqrt{\tilde{\beta}^{2}-4 \tilde{\alpha}}\right) / 2$.

3. $\left|g\left(\theta_{1}\right)\right|=\left|g\left(\theta_{2}\right)\right|$.

4. $|g(0)|=\left|g\left(\theta_{i}\right)\right|$.

The first condition easily leads to $\tilde{\alpha}=-2$, see statement $(2 \mathrm{~d})$ of the theorem.

Using that

$$
g\left(\theta_{i}\right)=\frac{1}{3 \sqrt{1+T_{i}^{2}}}\left(\tilde{\beta} T_{i}-2\right), \text { for } i=1,2,
$$

the second condition becomes $\tilde{\beta} T_{i}-2=0$ which can be expressed as

$$
(2+\tilde{\alpha}) \tilde{\beta}^{2}+4=0,
$$

see also statement $(2 \mathrm{~d})$;

Again by (14), the condition $\left|g\left(\theta_{1}\right)\right|=\left|g\left(\theta_{2}\right)\right|$ can be reduced to:

$$
\tilde{\beta}\left(4 \tilde{\alpha}-\tilde{\beta}^{2}\right)=0 \text {. }
$$

Then, $\tilde{\beta}=0$ contributes to statement (2c) while $\Delta=\tilde{\beta}^{2}-4 \tilde{\alpha}=0$ is not really a saddle-connection curve, since on it $\theta_{1}=\theta_{2}$. In fact, it belongs to a critical point with only two hyperbolic sectors, as it is explained in statement (2e). Similarly, when $\tilde{\alpha}=0$, one of these critical points collides with the origin, and we have the same type of bifurcation.

On the other hand, still using (14), $|g(0)|=\left|g\left(\theta_{i}\right)\right|$ writes as

$$
T_{i}^{2}\left(\tilde{\beta}^{2}-\tilde{\alpha}^{2}-4 \tilde{\alpha}\right)-\tilde{\alpha}^{2}=0,
$$

or, equivalently, as the expression given in statements (2b) and (2c).

The rest of situations (statements (1) and (2a)) belong to the case $\gamma=0$, and follow more easily.

The bifurcation diagram given in Fig. 8 contents the projections of the curves described in Theorem 3 on the half-sphere $\alpha^{2}+\beta^{2}+\gamma^{2}=1, \gamma \geq 0$. Of course, the bifurcation curves of case $\gamma=0$ appear in this diagram as single points on the boundary. 


\subsection{The Cubic Case.}

In the following section we first give a complete classification of the phase portraits that can appear when a Hamiltonian with a quartic nonlinearity is considered. Eventually, we have included the study of the bifurcaton diagram for reversible systems.

\subsubsection{Classification of phase portraits}

A straightforward application of our techniques gives the following result for general cubic systems:

Theorem 4 The phase portrait of any Hamiltonian system of type (1) with $n=3$ is topologically equivalent to one of the 23 configurations given in Fig. 9.

The classification has been obtained from the different possible shapes of $g(\theta)$ and applying the above remarks, up to rotations and the simetry given by $\theta \rightarrow \pi-\theta$.

\subsubsection{Bifurcation diagram of cubic reversible systems.}

Definition 3 A planar system of differential equations is called to be a reversible system if is invariant under a rotation and a reparametrization of the time given by $\tilde{t}=-t$.

A straightforward computation shows that a reversible cubic hamiltonian system of type (1), arise from a hamiltonian of the form

$$
H(x, y)=\frac{x^{2}+y^{2}}{2}+a x^{4}+b x^{2} y^{2}+c y^{4}
$$

Following a procedure with the same spirit than in Section 4.1.2, we have the following result.

Theorem 5 Consider the Hamiltonian systems which Hamiltonian function is $H_{4}(r, \theta)=\frac{1}{2} r^{2}+r^{4} g(\theta)$. Suppose that $g(\theta)=a \cos ^{4} \theta+b \cos ^{2} \theta \sin ^{2} \theta+c \sin ^{4} \theta$, and $a, b, c \in \mathbb{R}$, satisfying $a^{2}+b^{2}+c^{2} \neq 0$. When $b>0$, define $\tilde{a}=a / b, \tilde{c}=c / b$. When $b<0$, define $\bar{a}=a / b, \bar{c}=c / b$.

Then, there appear bifurcations of codimension at most two inside this family of systems. More precisely, in the space $(a, b, c)$ : 
1. The codimension two bifurcation points are the intersections of the codimension one curves, and are the following (see Fig. 9): $(\tilde{a}, \tilde{c})=(0,0)$ $($ Cubic-4); $(\tilde{a}, \tilde{c})=(-1 / 2,-1 / 2) \quad($ Cubic-15); $(\bar{a}, \bar{c})=(1 / 2,1 / 2) \quad$ (Cubic-4); $(\bar{a}, \bar{c})=(0,0) \quad($ Cubic-15); $(\bar{a}, \bar{c})=(0,-1 / 2),(\bar{a}, \bar{c})=(-1 / 2,0) \quad($ Cubic- 6$) ;$ $(\bar{a}, \bar{c})=(-1 / 2,-1 / 2)($ Cubic-1); $a=b=0, c \neq 0$ (Cubic-3 if $c>0$ and Cubic- 6 if $c<0) ; c=b=0, a \neq 0$ (Cubic-3 if $a>0$ and Cubic-6 if $a<0)$.

2. The codimension one curves are (except for the codimension two points stated above):

(a) $a=c, b=0, a<0, c<0 \quad(C u b i c-12)$

(b) $\tilde{a}=0$ (Cubic-3 if $\tilde{c}>0$ and Cubic- 7 if $\tilde{c}<0), \tilde{c}=0$ (Cubic-3 if $\tilde{a}>0$ and Cubic- 7 if $\tilde{a}<0)$.

(c) $\tilde{a}=\tilde{c}, \tilde{a}<0, \tilde{c}<0$ (Cubic-14 if $\tilde{c}>-1 / 2$ and Cubic-12 if $\tilde{c}<-1 / 2$ ).

(d) $\tilde{c}=1 /(4 \tilde{a}), \tilde{a}<0, \tilde{c}<0$ (Cubic-9).

(e) $\bar{a}=0$ (Cubic-10 if $\bar{c}>0$, Cubic-19 if $\bar{c} \in(-1 / 2,0)$, Cubic-6 if $\bar{c}<-1 / 2$ ), $\bar{c}=0$ (Cubic-10 if $\bar{a}>0$, Cubic-19 if $\bar{a} \in(-1 / 2,0)$, Cubic-6 if $\bar{a}<-1 / 2)$.

(f) $\bar{a}=\bar{c}, \bar{a}<0, \bar{c}<0$ (Cubic-12).

(g) $\bar{a}=-1 / 2, \bar{c}<0$ or $\bar{c}=-1 / 2, \bar{a}<0$ (Cubic-11).

(h) $\bar{a}=-1 / 2, \bar{c}>0$ or $\bar{c}=-1 / 2, \bar{a}>0$ (Cubic-5).

(i) $\bar{c}=1 /(4 \bar{a}), \bar{a}>0, \bar{c}>0($ Cubic-4).

3. The rest of points in the bifurcation space correspond to structurally stable systems. In particular, the systems are topologically equivalent to Cubic- $j$, with

(a) $j=2$, if $b=0, a>0, c>0$.

(b) $j=5$, if $b=0, a \cdot c<0$.

(c) $j=13$, if $b=0, a<0, c<0, a \neq c$.

(d) $j=2$, if $\tilde{a}>0, \tilde{c}>0$.

(e) $j=5$, if $\tilde{a} \cdot \tilde{c}<0$.

(f) $j=8$, if $\tilde{c}>1 /(4 \tilde{a}), \tilde{a}<0, \tilde{c}<0, \tilde{a} \neq \tilde{c}$.

(g) $j=13$, if $\tilde{c}<1 /(4 \tilde{a}), \tilde{a}<0, \tilde{c}<0, \tilde{a} \neq \tilde{c}$.

(h) $j=2$, if $\bar{c}>1 /(4 \bar{a}), \bar{a}>0, \bar{c}>0$.

(i) $j=14$, if $\bar{c}<1 /(4 \bar{a}), \bar{a}>0, \bar{c}>0$.

(j) $j=18$, if $\bar{a}>0, \bar{c} \in(-1 / 2,0)$, or $\bar{c}>0, \bar{a} \in(-1 / 2,0)$.

(k) $j=12$, if $(\bar{a}, \bar{c}) \in(-1 / 2,0) \times(-1 / 2,0), \bar{a} \neq \bar{c}$.

(l) $j=5$, if $\bar{a}>0, \bar{c}<-1 / 2$ or $\bar{c}>0, \bar{a}<-1 / 2$. 


$$
\begin{aligned}
& \text { (m) } j=11 \text {, if } \bar{a}<-1 / 2, \bar{c} \in(-1 / 2,0) \text {, or } \bar{c}<-1 / 2, \bar{a} \in(-1 / 2,0) \text {. } \\
& \text { (n) } j=13 \text {, if } \bar{a}<-1 / 2, \bar{c}<-1 / 2, \bar{a} \neq \bar{c} .
\end{aligned}
$$

Proof of Theorem 5: The main step consists again of finding the codimension one curves. First, we must consider that always $\theta=0$ and $\theta=\pi / 2$ are critical points of $g$, although there can also appear two other critical points of $g$ given by

$$
\tan \theta_{i}=(-1)^{i+1} \sqrt{\frac{2 a-\operatorname{sign}(b)}{2 c-\operatorname{sign}(b)}} .
$$

First of all we must determine when $\theta_{1}$ and $\theta_{2}$ are defined, that is, when

$$
\frac{2 a-\operatorname{sign}(b)}{2 c-\operatorname{sign}(b)}>0
$$

In that case it is easy to check that $g\left(\theta_{1}\right)=g\left(\theta_{2}\right)$.

Taking into account these previous considerations, we obtain the codimension one curves by solving the following equations:

1. $g(0)=0$.

2. $g(\pi / 2)=0$.

3. $g\left(\theta_{1}\right)=g\left(\theta_{2}\right)=0$.

4. $g(0)=g\left(\theta_{1}\right)=g\left(\theta_{2}\right)$.

5. $g(\pi / 2)=g\left(\theta_{1}\right)=g\left(\theta_{2}\right)$.

6. $g(0)=g(\pi / 2)$.

We present the bifurcation diagram compactified on the sphere $a^{2}+b^{2}+c^{2}=1$; We only give the sketch of the proof for the half-sphere determined by $b>0$. When $b=0$ or $b<0$, the proof can be obtained analogously.

Using that $g(0)=\tilde{a}, g(\pi / 2)=\tilde{c}$, and $g\left(\theta_{1}\right)=g\left(\theta_{2}\right)=(-1+4 \tilde{a} \tilde{c}) /(4(-1+\tilde{a}+\tilde{c}))$, we easily obtain that the first, second and third conditions lead to $\tilde{a}=0, \tilde{c}=0$ and $\tilde{c}=1 /(4 \tilde{a})$, respectively (see statements $(2 \mathrm{~b})$ and $(2 \mathrm{~d})$ ). The fourth and fifth conditions lead to $\tilde{a}=1 / 2$ and $\tilde{c}=1 / 2$ but in these cases $\theta_{1}$ and $\theta_{2}$ are not defined. Finally, the sixth condition leads to $\tilde{a}=\tilde{c}$ (see statement $(2 \mathrm{c})$ ).

The bifurcation diagram contents the projections of the curves described in Theorem 5 on the half-spheres of $a^{2}+b^{2}+c^{2}=1$ defined by $b \geq 0$ and $b \leq 0$. The bifurcation curves of the case $b=0$ appear in these diagrams as single points on the boundary. 


\section{Appendix}

Theorem 6 (Nilpotent critical point theorem [20]) Let $F$ and $G$ be analytic functions in a neighbourhood of the origin starting with quadratic terms in the variables $x$ and $y$. Let $(0,0)$ be an isolated singular point of the vector field $(y+F(x, y), G(x, y))$, and let $y=f(x)$ be the solution of the equation $y+F(x, y)=0$ in a neighbourhood of $(0,0)$. Assume that the expansion of the function $G(x, f(x))$ is of the form $\Phi_{1}(x)=G(x, f(x))=K x^{\kappa}+o(\kappa)$ and $\Phi_{2}(x) \equiv(\partial F / \partial x+\partial G / \partial y)(x, f(x))=$ $L x^{\lambda}+o(\lambda)$ with $K \neq 0, \kappa \geq 2$ and $\lambda \geq 1$. Then the following statements hold.

1. If $\kappa$ is even and

(a) $\kappa>2 \lambda+1$, then the origin is a saddle-node. Moreover the saddle-node has the three separatrices tangent to the $x$-axis, two of them tangent to one half-axis and the third one tangent to the other half-axis, and all the orbits of the nodal sectors are tangent to the $x$-half-axis having a unique tangent separatrix.

(b) $\kappa<2 \lambda+1$ or $\Phi_{2} \equiv 0$, then the origin is a cusp, i.e. a singular point formed by the union of two hyperbolic sectors. Moreover, the cusp has two separatrices tangent to the positive $x$-axis.

2. If $\kappa$ is odd and $K>0$, then the origin is a saddle. Moreover, the saddle has two separatrices tangent to the semi-axis $x<0$, and the other two tangent to the semi-axis $x>0$.

3. If $\kappa$ odd, $K<0$ and

(a) $\lambda$ even, $\kappa=2 \lambda+1$ and $L^{2}+4 K(\lambda+1) \geq 0$, or $\lambda$ even and $\kappa>2 \lambda+1$, then the origin is a stable (unstable) node if $L<0(L>0)$, having all the orbits tangent to the $x$-axis at $(0,0)$.

(b) $\lambda$ odd, $\kappa=2 \lambda+1$ and $L^{2}+4 K(\lambda+1) \geq 0$, or $\lambda$ odd and $\kappa>2 \lambda+1$, then the origin is an elliptic-saddle, i.e., a singular point formed by the union of one hyperbolic sector and one elliptic sector. Moreover, one separatrix of the elliptic-saddle is tangent to the semi-axis $x<0$, and the other to the semi-axis $x>0$.

(c) $\kappa=2 \lambda+1$ and $L^{2}+4 K(\lambda+1)<0$, or $\kappa<2 \lambda+1$, or $\Phi_{2}(x) \equiv 0$, then the origin is either a centre or a focus.

\section{References}

[1] Li Jibin, Huang Qiming. Bifurcations of limit cycles forming compound eyes in the cubic system, Chin. Ann. of Math. 8 Ser. B (1987), 391-403. 
[2] Gasull A., Guillamon A., Mañosa V., Mañosas F. The period function for Hamiltonian systems with homogeneous nonlinearities, J. of Diff. Eq. 139 1997, $237-260$.

[3] Rothe F. Remark on periods of planar Hamiltonian systems, SIAM J. of Math. Anal. 24 (1993), 129-154.

[4] Christopher C.J., Devlin J. Isochronous centres in planar polynomial systems, SIAM J. of Math. Anal. 28 (1997), 162-177.

[5] Schumann B. Sur la forme normale de Birkhoff et les centres isochrones, C.R. Acad. Sci. Paris. 322-I (1996), 21-24.

[6] Bass H., Connell E., Wright D. The Jacobian conjecture: reduction of degree and formal expansion of the inverse. Bull. of AMS. 7 (1982), 287-330.

[7] Sabatini M. A connection between isochronous Hamiltonian centres and the Jacobian conjecture, Preprint, Università degli Studi di Trento, UTM 460 (1995).

[8] Cima A., Gasull A., Mañosas F. On polynomial Hamiltonian Planar Vector Fields, J. of Diff. Eq. 106 (1993), 367-383.

[9] Conti R,. Centers of quadratic systems, Ricerche di Matematica Suppl. XXXVI (1987), 117-126.

[10] Artés J.C., Llibre J. Quadratic Hamiltonian Vector Fields, J. of Diff. Eq. 107 (1994), 80-95.

[11] Schlomiuk D. Basic algebro-geometric concepts in the study of planar polynomial vector fields, Pub. Matemàtiques 41 (1997), 269-295.

[12] Arnold V.I. Ordinary Differential Equations, Springer-Verlag, New York Heidelberg Berlin, 1992.

[13] Cairó L., Llibre J. Phase portraits of planar semi-homogeneous vector fields-I, Nonlinear Analysis, T.M.A. 29 (1997), 783-811.

[14] de Jäger P. Phase portraits for quadratic systems with a higher order singularity with two zero eigenvalues, J. of Diff. Eq. 87 (1990), 169-204.

[15] Gasull A., Prohens R. On quadratic systems with a degenerate critical point, Rocky Mount. J. Math. 26 (1996), 135-164.

[16] Reijn J.W. A bibliography of the qualitative theory of quadratic systems of differential equations in the plane, Third Edition, Delft Univ. of Technology, Report 94-02, 1994. 
[17] Blows T.R., Lloyd N.G.. The number of limit cycles of certain polynomial differential equations, Proc. of the Roy. Soc. of Edinburgh 98A (1984), 215-239.

[18] González E.A. Generic properties of polynomial vector fields at infinity, Trans. of AMS. 143 (1969), 201-222.

[19] Cherkas L.A. Number of limit cycles of an autonomous second-order system, Diff. Eq. 5 (1976), 666-668.

[20] Andreev A. Investigation of the behaviour of the integral curves of a system of two differential equations in the neighbourhood of a singular point, Translation of $A M S 8$ (1958), 187-207.

[21] Markus L. Global structure of ordinary differential equations in the plane, Trans. of AMS. 76 (1954), 127-148.

[22] Vulpe N.I. Affine invariant conditions for the topological discrimination of a quadratic systems with a centre, Diff. Eq. 19 (1983), 273-280. 

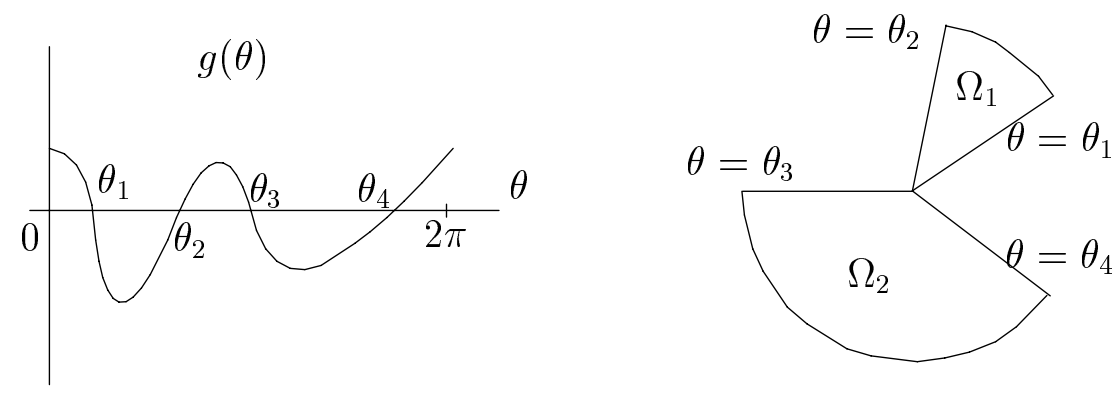

Figure 1: Two basic sectors defined by a concrete $g(\theta): \Omega_{1}$ and $\Omega_{2}$.

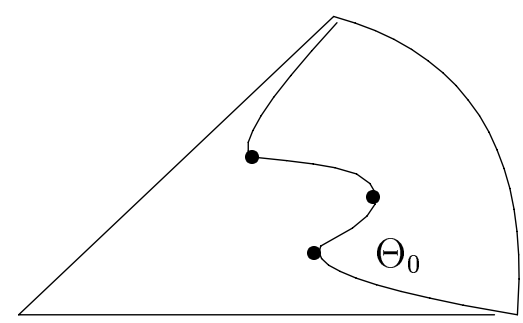

Figure 2: In a basic sector, the isocline $\Theta_{0}=\{(r, \theta): \dot{\theta}=0\}$ connects all the critical points (both finite and infinite) and can be parameterized by $\theta$.

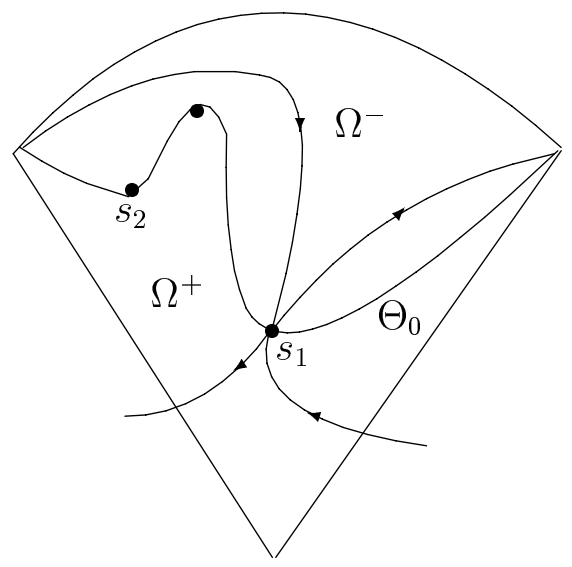

Figure 3: The saddle $s_{1}$ satisfies both properties $\left(\mathrm{LS}^{-}\right)$and $\left(\mathrm{LS}^{+}\right)$, while the other one, $s_{2}$, satisfies only $\left(\mathrm{LS}^{+}\right)$. 

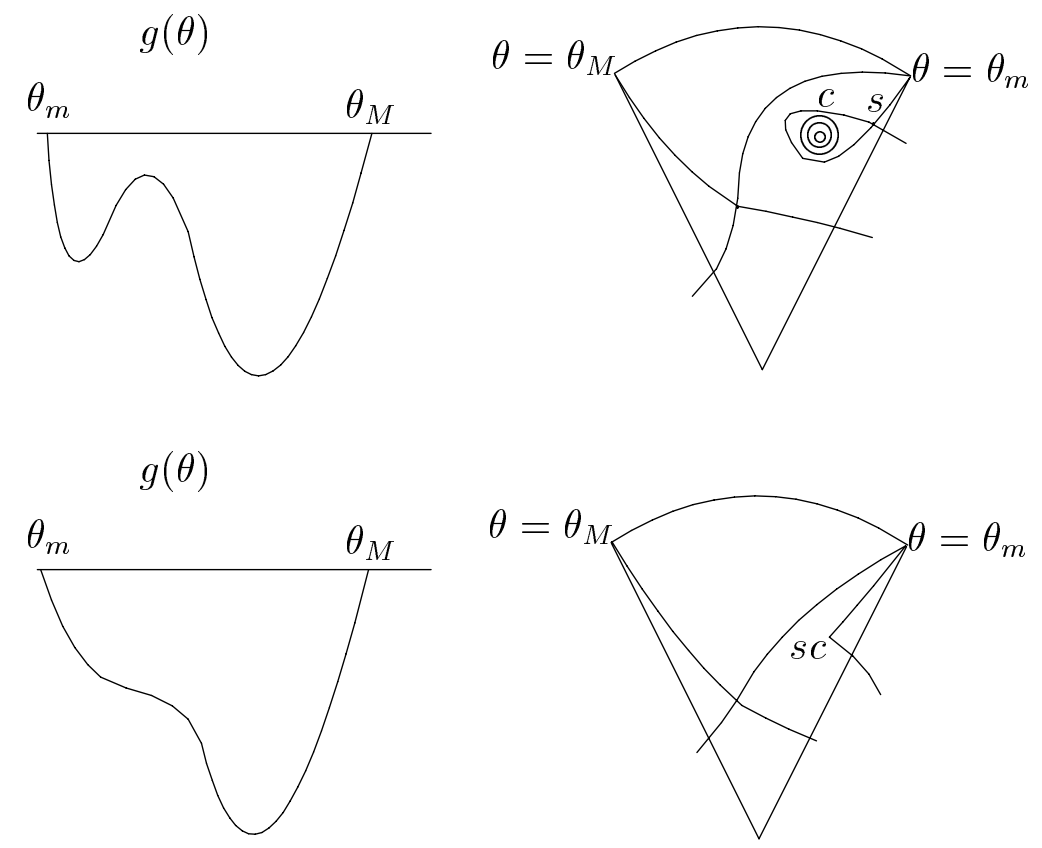

Figure 4: The transition from a saddle-centre configuration to a point with only two hyperbolic sectors
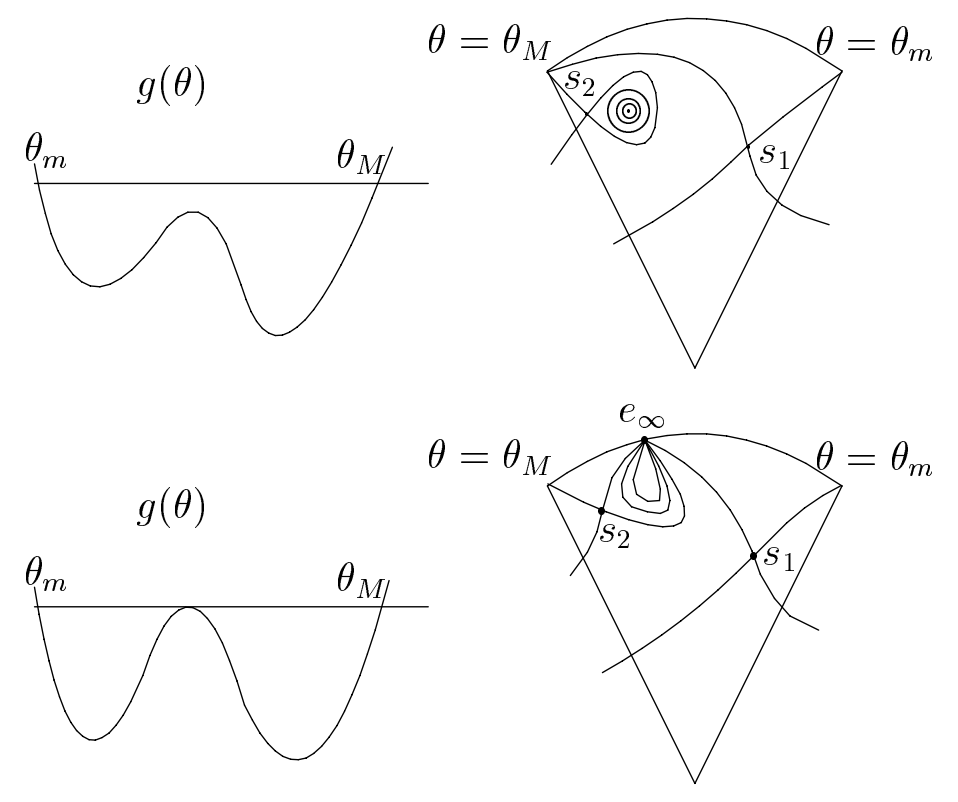

Figure 5: The transition from a finite centre to an elliptic sector at infinity. 
Figure 6: A phase portrait that arises from different shapes of $g$. 


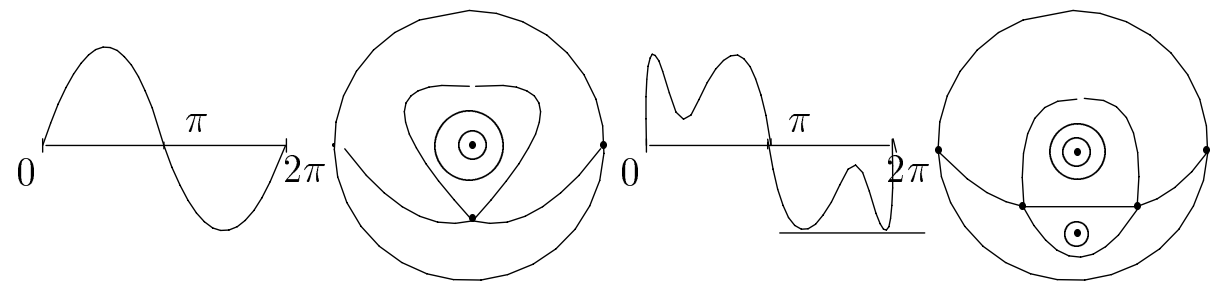

VULPE 2

VULPE 3

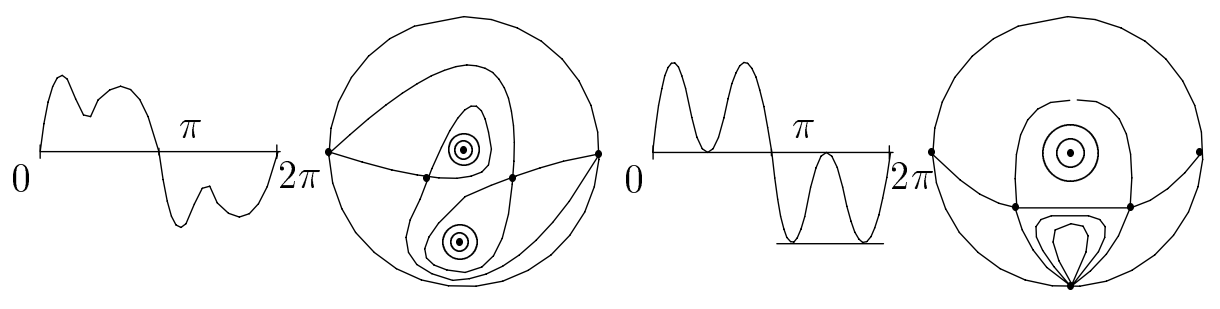

VULPE 4

VULPE 5

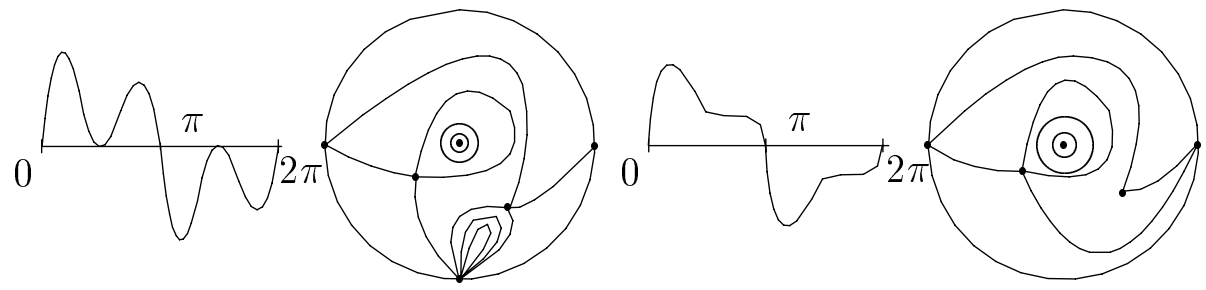

VULPE 6

VULPE 7

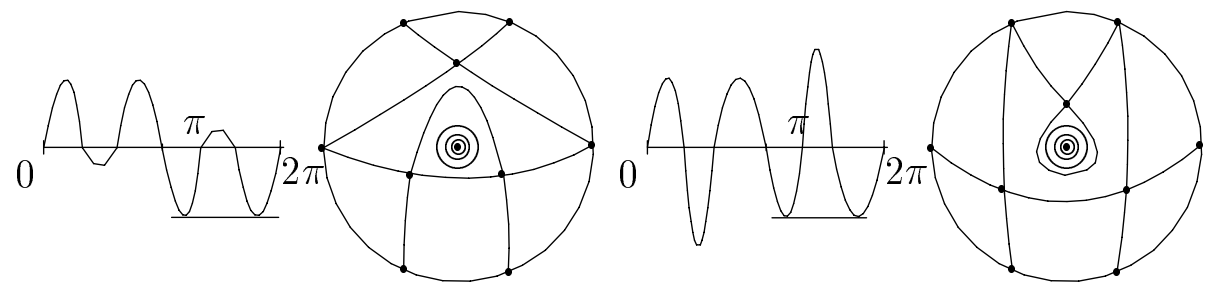

VULPE 8

VULPE 9

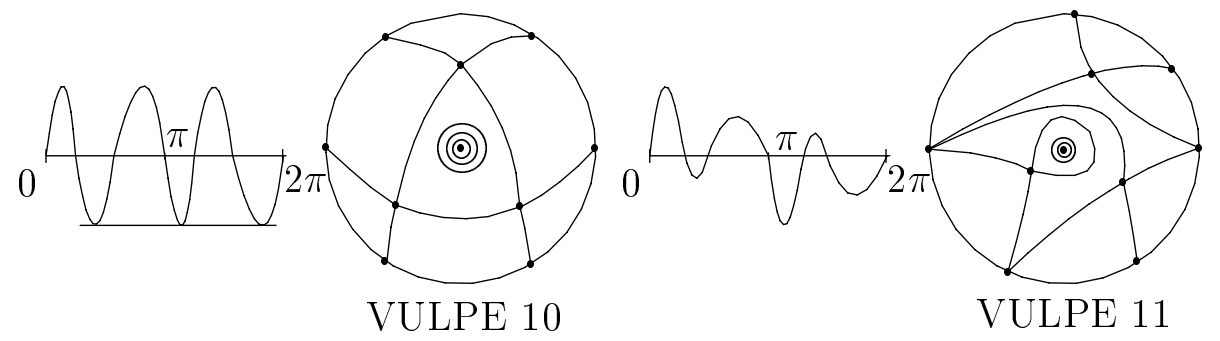

Figure 7: Phase portraits of quadratic Hamiltonian systems. 


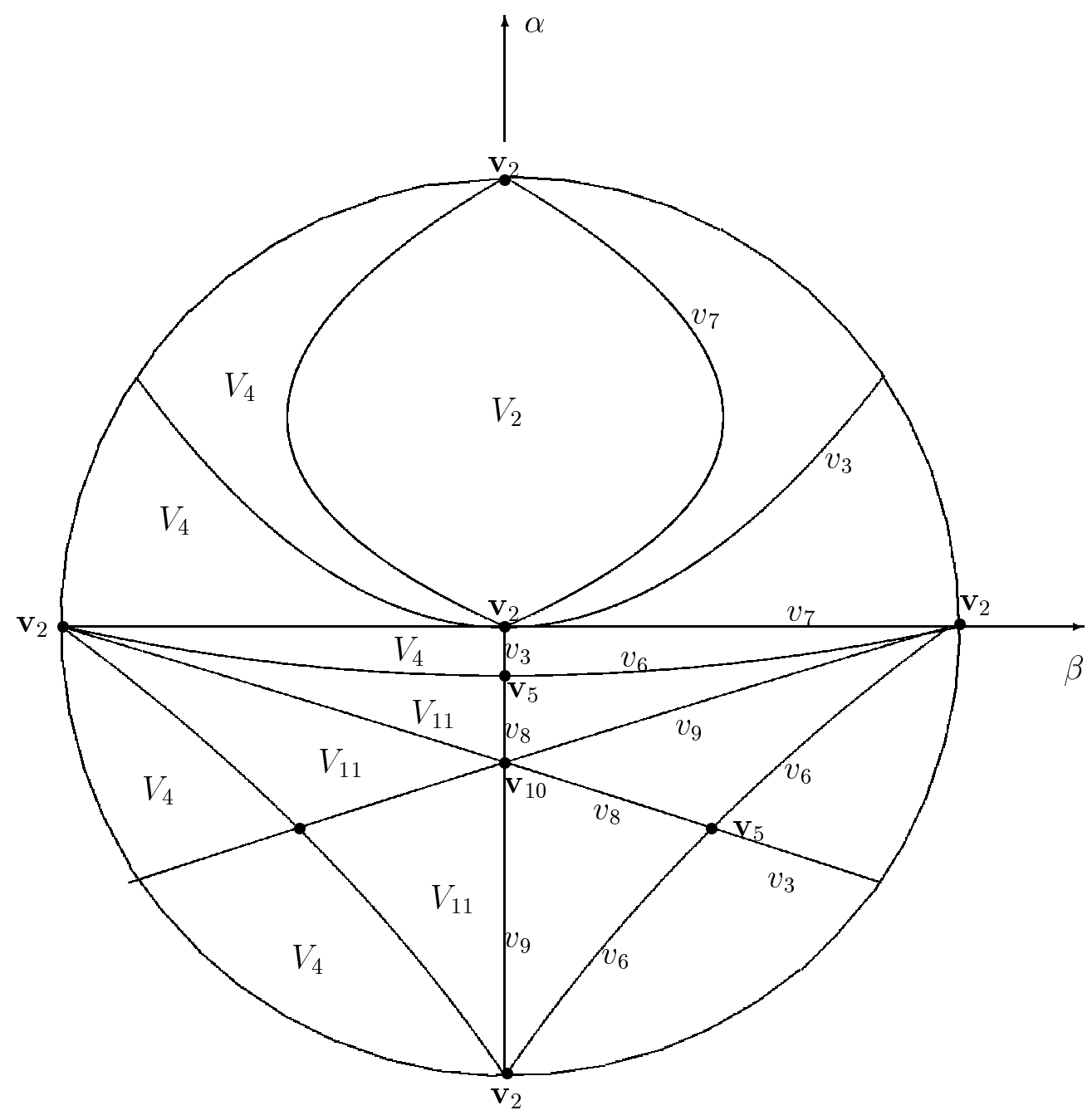

Figure 8: Bifurcation diagram on the sphere $\alpha^{2}+\beta^{2}+\gamma^{2}=1, \gamma \geq 0$; for $\gamma \leq 0$, the diagram is the same changing the sign of $\alpha$. Since it is a symmetric diagram and in order to clarify the display, we have marked on $\beta<0$ only the structurally stable systems, with the corresponding Vulpe type topological equivalence in capital letters, $V_{j}$, and, on $\beta>0$ only the unstructurally stable ones, with the equivalence in low italics letters, $v_{j}$, if they are of codimension one, and in bold letters, $\mathbf{v}_{\mathbf{j}}$, if they are of codimension two. 
Figure 9: Phase portraits of cubic Hamiltonian systems. 
Figure 10: Bifurcation diagram on the sphere $a^{2}+b^{2}+c^{2}=1, c \geq 0$ and $c \leq 0$. Since the diagrams are symmetric with respect the $\tilde{a}=\tilde{c}$ and $\bar{a}=\bar{c}$ lines, and in order to clarify the display, we have marked only in one side of the bifurcation diagrams the corresponding type of topological equivalence: the structurally stable systems, with the topological class of equivalence in capital letters, $C_{j}$; the structurally unstable ones, with the equivalence in low italics letters, $c_{j}$, if they are of codimension one, and in bold letters, $\mathbf{c}_{\mathbf{j}}$, if they are of codimension two. 